\title{
Efficient and Accurate Modeling of Electrically Large Dielectric Lens Antennas using Full-Wave Analysis
}

\author{
Stig B. Sørensen ${ }^{1}$, Min Zhou ${ }^{1}$, Peter Meincke ${ }^{1}$, Niall Tynan ${ }^{2}$, and Marcin L. Gradziel ${ }^{2}$ \\ ${ }^{1}$ TICRA, Copenhagen, Denmark, ticra@ticra.com \\ ${ }^{2}$ National University of Ireland, Maynooth, Ireland, niall.tynan.2012@nuim.ie
}

\begin{abstract}
Two efficient analysis methods for the accurate modeling of electrically large dielectric lens antennas are presented. The first method is based on Double Physical Optics (PO), which takes into account an additional set of reflections within the lens, whereas the conventional PO method only accounts for one set. The second method relies on a higher-order Body of Revolution Method of Moments and is capable of providing a full-wave solution of a 100 -wavelength dielectric lens antenna within 2 minutes on a laptop computer.
\end{abstract}

Index Terms-modeling, dielectric lens, higher-order MoM

\section{INTRODUCTION}

Dielectric lens antennas possess several advantages compared to reflector antennas, e.g., enhanced wide-scan capability, no blockage, ease of fabrication due to their rotational symmetry, greater flexibility, etc. [1]. Dielectric lenses have been suggested as an alternative solution to an offset dual reflector system for scientific space applications [2]. Also, they are easy to design using Geometrical Optics (GO) or Physical Optics (PO). However, they are challenging from an EM modeling point of view, since they are electrically large and often coated with matching layers. High-frequency methods, e.g. conventional PO, do not accurately account for the reflections inside the lens. One way to alleviate this is by applying a Double PO approach where an additional set of reflections within the lens is taken into account. To check the validity of such an approach, one needs to employ full-wave analysis tools which includes multiple interactions of all orders within the lens. However, due to the large electrical size, the typical computation time using existing commercial tools is in terms of several hours.

In this paper, the Double PO method is described and validated by full-wave analysis based on the Body of Revolution Method of Moments (BoR-MoM), which is commercially available in TICRA's software package GRASP [3]. The two methods are applied to large dielectric lens antennas and validated by comparison with measurements.

\section{AnALysis Methods}

\section{A. Double Physical Optics}

Initially, the incident field due to the feed is used to find the equivalent electric and magnetic surface current densities on the front surface of the lens,

$$
\vec{J}_{s f}=\hat{n} \times \vec{H}, \quad \vec{M}_{s f}=-\hat{n} \times \vec{E}
$$

where $\hat{n}$ is the outward normal unit vector, and $\vec{H}, \vec{E}$ denote the total magnetic and electric fields, respectively. At any point on the front surface the equivalent currents can be computed using the Fresnel reflection and transmission coefficients for plane-wave incidence on a infinite planar dielectric interface. The direction of incidence is determined by the Poynting vector such that the locally reflected and transmitted field can be computed [4]. When these fields are known the equivalent surface current densities follow directly from (1). By letting $\vec{J}_{s f}, \vec{M}_{s f}$ radiate in the dielectric lens material, the incident field on the back surface of the lens is computed, so that a set of equivalent surface current densities $\vec{J}_{s b}, \vec{M}_{s b}$ on this surface is found. Again, the Poynting vector and the Fresnel reflection and transmission coefficients are used. The currents on the back surface are subsequently used to calculate a new set of currents on the front surface, from which a new set of currents is determined on the back. The radiation from the currents constitutes the field scattered by the lens. Hence, the difference between conventional PO and Double PO is that the latter method includes an additional set of reflections within the lens.

\section{B. Higher-Order Body of Revolution Method of Moments}

The BoR-MoM has been formulated previously in several works, e.g., [5], [6]. These works employed triangular basis functions on linear curve segments, requiring a relatively large number of unknowns to achieve convergence. We employ the same integral equation as in previous works, e.g., that of [6], but the equation is discretized with higher-order basis functions and curvilinear segments. The electric and magnetic surface current densities, $\vec{J}_{s}$ and $\vec{M}_{s}$, on each curve segment are expanded as

$$
\vec{X}=\sum_{m=0}^{M^{\phi}} \vec{X}_{m}
$$


where $\vec{X}=\vec{J}_{s}, \vec{M}_{s}$ and

$$
\begin{aligned}
\vec{X}_{m} & =\sum_{n=0}^{N^{t}} a_{m n}^{t, e} \vec{B}_{m n}^{t, e}+a_{m n}^{t, o} \vec{B}_{m n}^{t, o} \\
& +\sum_{n=0}^{N^{t}-1} a_{m n}^{\phi, e} \vec{B}_{m n}^{\phi, e}+a_{m n}^{\phi, o} \vec{B}_{m n}^{\phi, o} .
\end{aligned}
$$

Herein, $a_{m n}^{t, e}, a_{m n}^{t, o}, a_{m n}^{\phi, e}$, and $a_{m n}^{\phi, o}$ are unknown coefficients, $N^{t}$ is the polynomial expansion order along the generatrix, $M^{\phi}$ is the highest azimuthal mode index, and $\vec{B}_{m n}^{t, e}, \vec{B}_{m n}^{t, o}$, $\vec{B}_{m n}^{\phi, e}$, and $\vec{B}_{m n}^{\phi, o}$ are $t$ - and $\phi$-directed vector basis function, defined as

$$
\begin{aligned}
& \vec{B}_{m n}^{t,\left(\begin{array}{c}
e \\
o
\end{array}\right)}(t, \phi)=\frac{\vec{a}_{t}}{\mathcal{J}_{s}(t, \phi)} \widetilde{P}_{n}(t)\left(\begin{array}{c}
\cos m \phi \\
\sin m \phi
\end{array}\right), \\
& \vec{B}_{m n}^{\phi,\left(c_{o}^{e}\right)}(t, \phi)=\frac{\vec{a}_{\phi}}{\mathcal{J}_{s}(t, \phi)} P_{n}(t)\left(\begin{array}{c}
\cos m \phi \\
\sin m \phi
\end{array}\right) .
\end{aligned}
$$

Here, $\vec{a}_{t}=\partial \vec{r} / \partial t, \vec{a}_{\phi}=\partial \vec{r} / \partial \phi$, are the covariant unitary vectors, and $\mathcal{J}_{s}(t, \phi)=\left|\vec{a}_{t} \times \vec{a}_{\phi}\right|$. In (4b) the polynomials $P_{n}(t)$ along the direction transverse to the current flow are the Legendre polynomials due to their orthogonality properties. In the direction along the current flow in (4a), the modified Legendre polynomials

$$
\widetilde{P}_{n}(t)= \begin{cases}1-t, & n=0 \\ 1+t, & n=1 \\ P_{n}(t)-P_{n-2}(t), & n \geq 2\end{cases}
$$

are used. The two lowest order polynomials can be matched with the corresponding functions on the neighbouring segments, or alternatively, they can be left out at external nodes.

The expansion order along the generatrix, $N^{t}$, is adapted to the electrical length of each segment, which is usually in the order of $2 \lambda, \lambda$ being the wavelength of the scatterer. The highest azimuthal mode index, $M^{\phi}$, is determined automatically from the Fourier series expansion of the incident field.

When higher-order basis functions and curved segments are used, the number of unknowns is reduced by a factor of 4 and drops to about 3-4 per wavelength. Consequently, even structures larger than several hundred wavelengths can be handled with modest computer resources.

The solutions for different azimuthal modes do not couple and therefore, the MoM problem for the unknowns $a_{m n}^{t, e}, a_{m n}^{t, o}$, $a_{m n}^{\phi, e}$, and $a_{m n}^{\phi, o}$ decouples into $M^{\phi}+1$ independent smaller MoM problems. Hence, to obtain the solution to the unknown surface current densities, a MoM problem - based on the expansion (3) - is solved for each azimuthal index, and the resulting solutions are summed according to (2).

\section{ApPLICATION EXAMPLE}

The methods described above are applied to the type B lens from [2]. This type B lens transforms rays from a point source into a planar wavefront, as shown in Figure 1. The lens has a diameter of $286 \mathrm{~mm}$ and is tested in the frequency range from 75 to $150 \mathrm{GHz}$. Thus, the dimension of the lens spans from $72-143 \lambda_{0}$, with $\lambda_{0}$ being the free-space wavelength.
The relative permittivity varies in the frequency range and was measured to be approximately $\epsilon_{r}=2.3$ at $100 \mathrm{GHz}$. Hence, the diameter of the lens is $145 \lambda$ at $100 \mathrm{GHz}$, where $\lambda$ is the wavelength of the dielectric. The centre of the lens is located at $z=410.5 \mathrm{~mm}$. The feed is a corrugated horn.

In Section III-A the feed is located on the axis of symmetry, whereas an offset feed is considered in Section III-B.

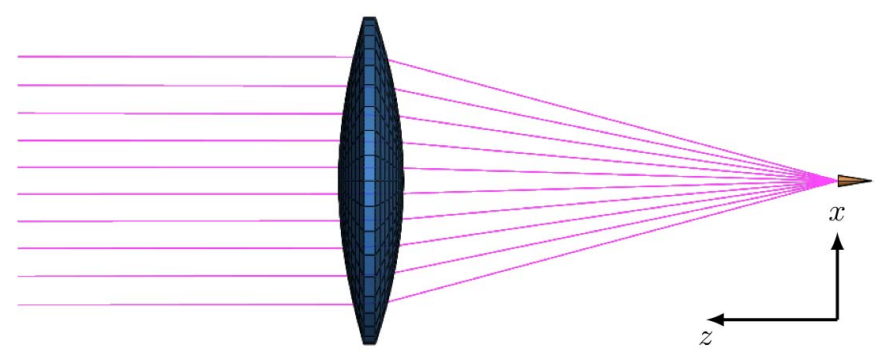

Fig. 1. The dielectric lens with on-axis illumination.

\section{A. On-axis Illumination}

The corrugated horn aperture is at $z=0 \mathrm{~mm}$. The amplitudes of the co- and cx-polar components of the radiated field at $100 \mathrm{GHz}$, calculated in the aperture plane at $z=510 \mathrm{~mm}$, are shown in Figures 2 and 3, respectively. The conventional PO, Double PO, and BoR-MoM are used to calculate the radiation patterns. Since the feed is a corrugated horn located at the axis of symmetry, only one azimuthal mode is needed in the BoR-MoM analysis.

It is seen that the overall agreement between the three methods for the co-polar component is good. However, there are ripples in the pattern - arising from the reflections within the lens - which are only predicted by BoR-MoM and Double PO. Furthermore, it is also observed that the cx-polar radiation obtained by conventional PO deviates somewhat from the solutions found using Double PO and BoR-MoM.

The computation time on a $2.6 \mathrm{GHz}$ Intel Core i7 laptop is approximately $30 \mathrm{~s}, 10 \mathrm{~min}$, and $1.5 \mathrm{~min}$ for PO, Double PO, and BoR-MoM, respectively. Despite BoR-MoM being a fullwave method, the computation time is very low and much lower compared to that of Double PO.

The simulated radiation pattern at $100 \mathrm{GHz}$ is compared with measurements in Figure 4. There is excellent agreement between simulations and measurements. The ripples predicted by the BoR-MoM analysis cannot be captured by the measurements due to insufficient measurement points.

\section{B. Offset Illumination}

Figure 5 depicts the configuration involving the offset illumination. The lens and feed are the same as in the previously considered case, but the lens is rotated 10 degrees around its center in the $x z$-plane. Consequently, the feed is located $410.5 \mathrm{~mm} \cdot \sin 10^{\circ}=71.3 \mathrm{~mm}$ from the symmetry axis of the lens, corresponding to $23.8 \lambda_{0}$. For this offset, 58 azimuthal modes $\left(M^{\phi}=57\right)$ are needed in the BoR-MoM analysis, and the calculation time increases to 1.5 hours. The computation 


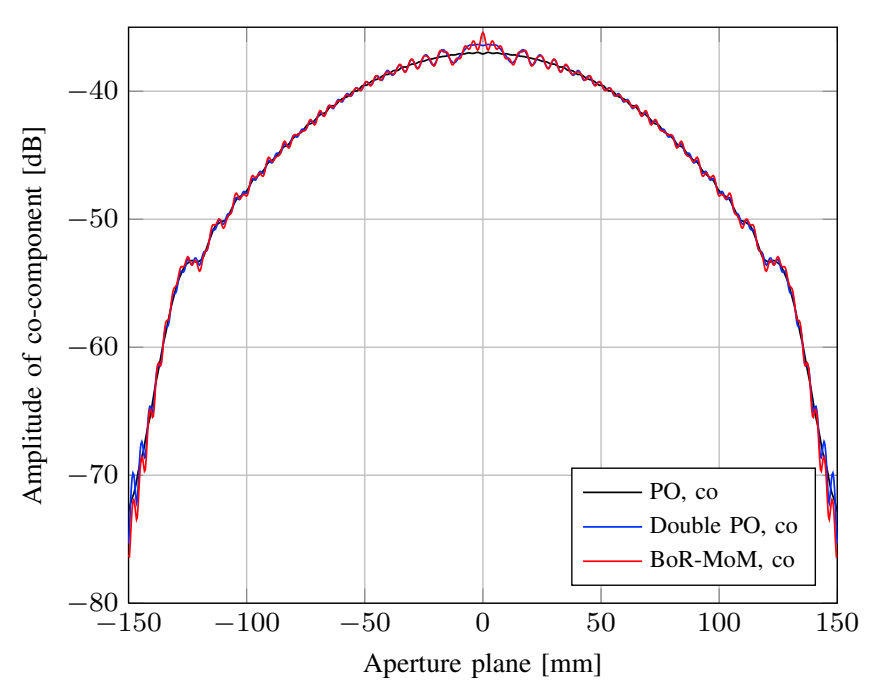

Fig. 2. The amplitude of the co-component of the radiated field at $\phi=45^{\circ}$ obtained by PO (black), Double PO (blue), and BoR-MoM (red) for the onaxis illumination case.

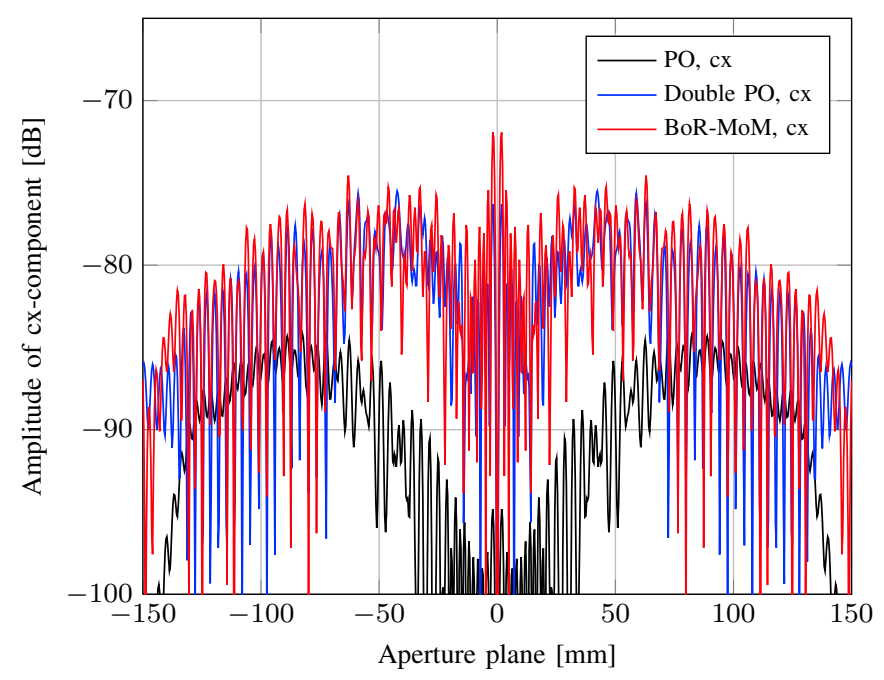

Fig. 3. The amplitude of the cx-component of the radiated field at $\phi=45^{\circ}$ obtained by PO (black), Double PO (blue), and BoR-MoM (red) for the onaxis illumination case.

time for Double PO remains the same, that is, approximately $10 \mathrm{~min}$, so for this offset case, Double PO is significantly less time consuming than BoR-MoM.

Figure 6 shows the co-polar component of the radiated field in the aperture plane $z=510 \mathrm{~mm}$, calculated by the three considered analysis methods. As for the on-axis illumination case it is observed that Double PO accurately accounts for the reflections within the lens, since the ripple in the patterns compare extremely well with those predicted by BoR-MoM. Further, the comparison between the cx-polar component of the radiated field, as shown in Figure 7, reveals that Double PO does not compare as well with BoR-MoM as for the on-axis illumination case, but still significantly better than conventional PO. Finally, Figure 8 shows that measurements compare well with the BoR-MoM results.

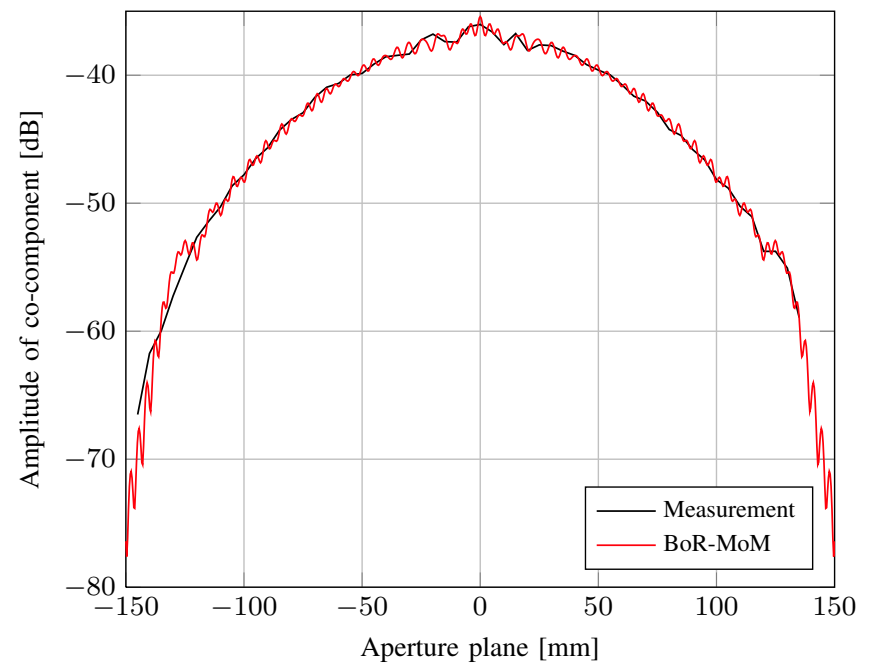

(a)

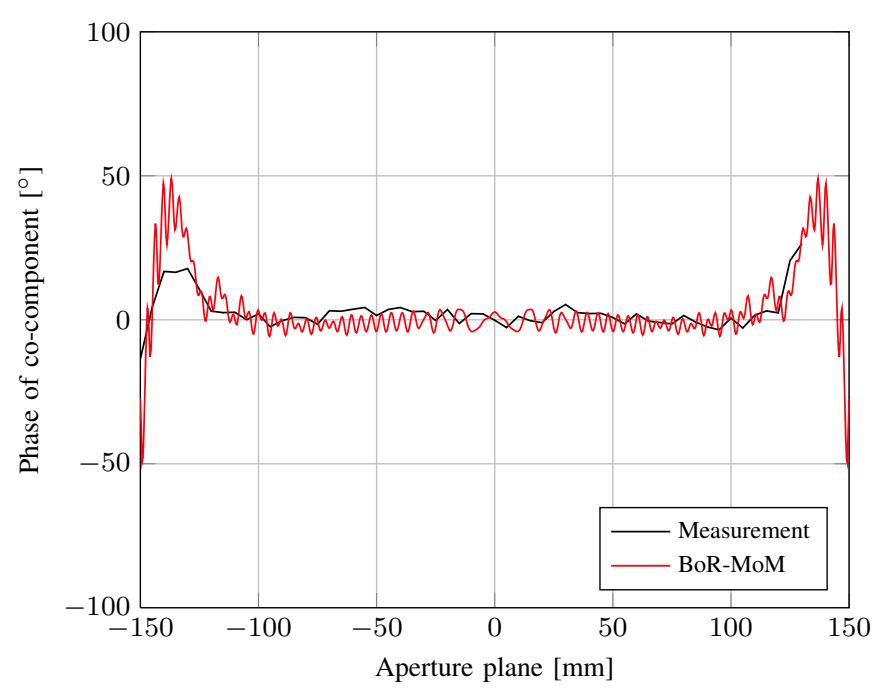

(b)

Fig. 4. The (a) amplitude and (b) phase of the co-component of the radiated field at $\phi=0^{\circ}$ obtained by measurements (black) and BoR-MoM (red) for the on-axis illumination case.

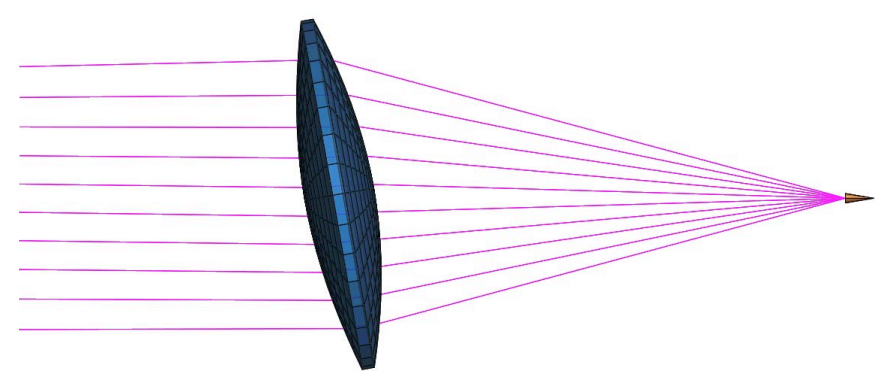

Fig. 5. The dielectric lens with offset illumination.

\section{CONCLUSiOnS}

Two methods for efficient and accurate analysis of electrically large dielectric lens antennas were presented, and comparisons with measurements were performed. First, it was shown that the derived higher-order BoR-MoM is capable of 


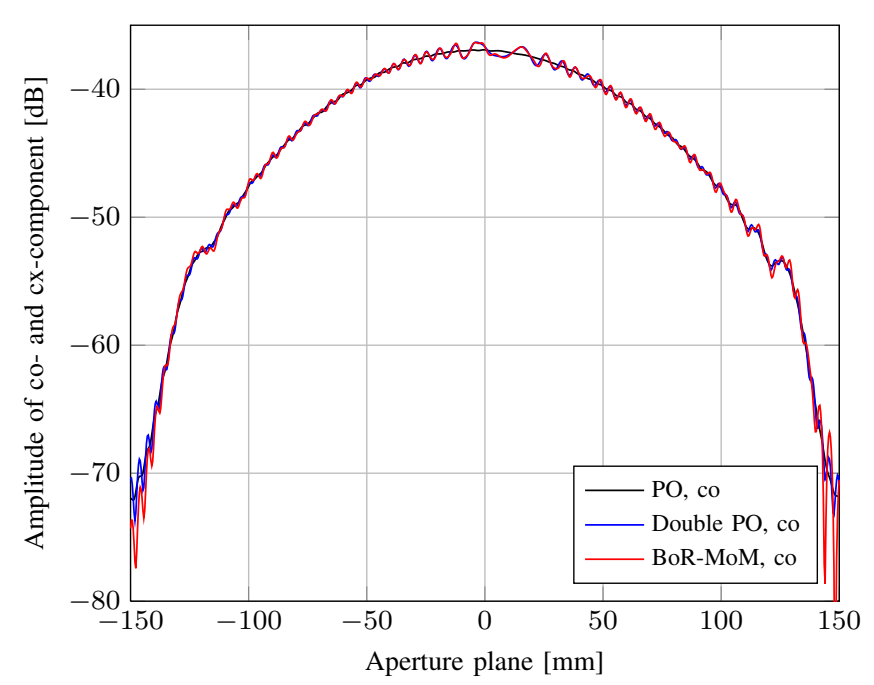

Fig. 6. The amplitude of the co-component of the radiated field at $\phi=45^{\circ}$ obtained by PO (black), Double PO (blue), and BoR-MoM (red) for the offsetillumination case.

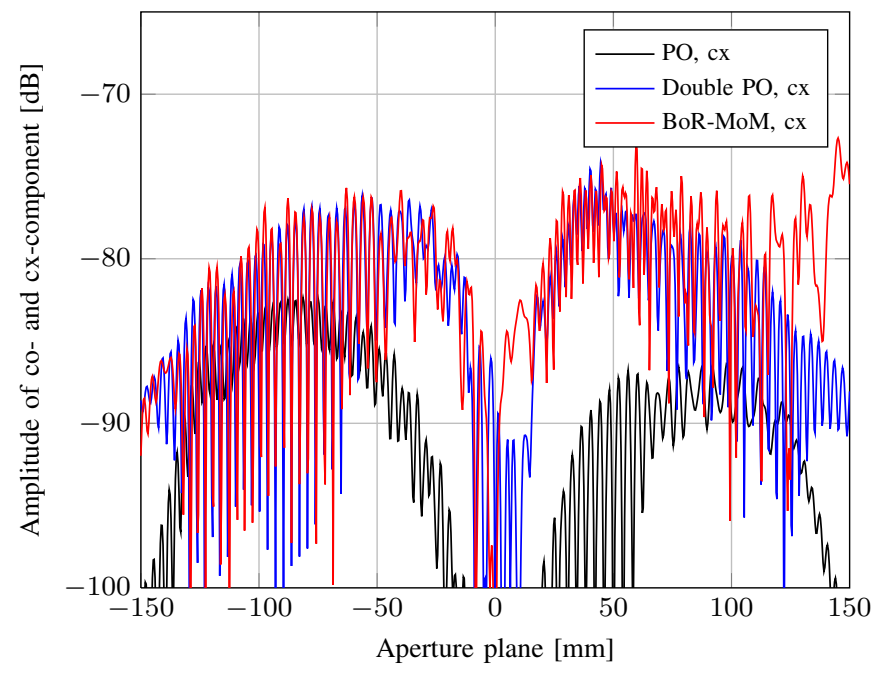

Fig. 7. The amplitude of the cx-component of the radiated field at $\phi=45^{\circ}$ obtained by PO (black), Double PO (blue), and BoR-MoM (red) for the offsetillumination case.

analyzing an electrically large lens with on-axis illumination (one azimuthal mode only) in few minutes on a standard laptop. The analysis time increases proportionally when more azimuthal modes are needed, which is the case for offset feed illumination. Second, it was shown that the asymptotic Double PO method, that takes into account an additional set of reflections inside the lens as compared to traditional PO, gives extremely accurate results for the co-polar component of the radiated field, when compared to BoR-MoM, and accurate results for the cx-polar component. Finally, we showed excellent agreement between BoR-MoM simulations and measurements of a 100 wavelength dielectric lens.

\section{ACKNOWLEDGMENT}

This work is supported by the European Space Agency under contract number 4000102522/10/NL/AF2011.

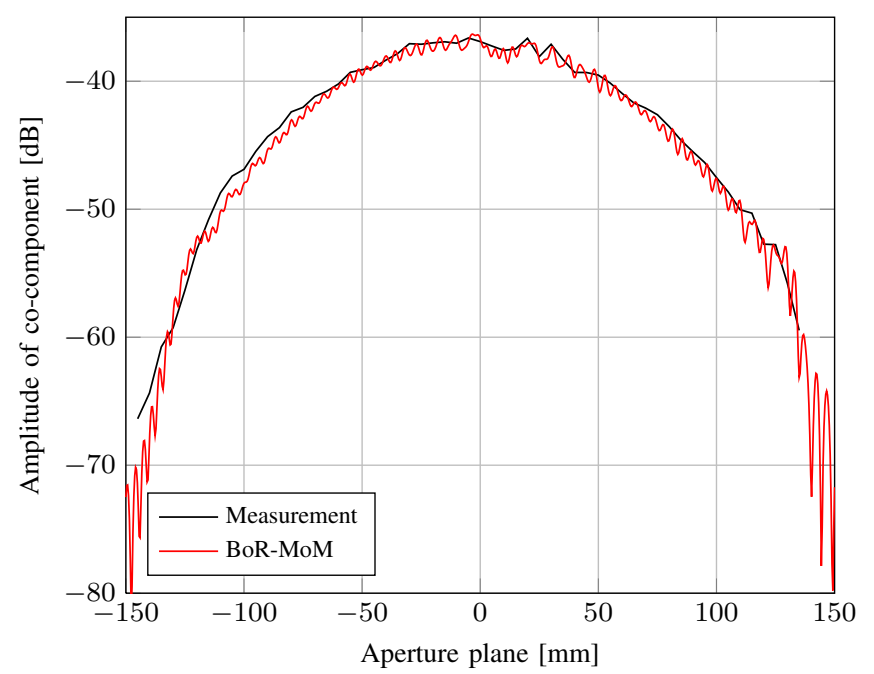

(a)

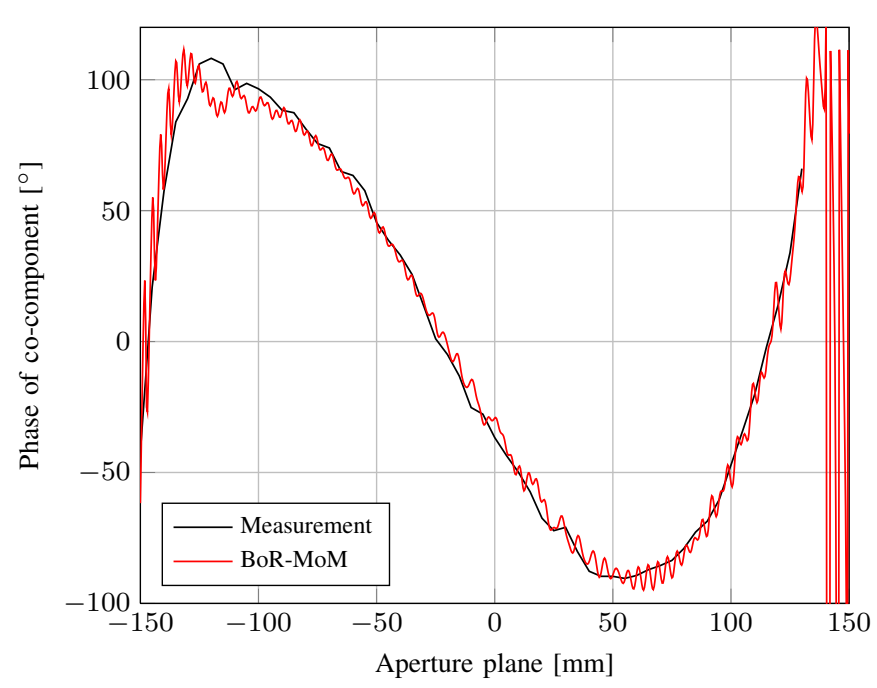

(b)

Fig. 8. The (a) amplitude and (b) phase of the co-component of the radiated field at $\phi=0^{\circ}$ obtained by measurements (black) and BoR-MoM (red) for the offset-illumination case.

\section{REFERENCES}

[1] R. C. Johnson and H. Jasik, Eds., Antenna Engineering Handbook. McGraw-Hill, 1961.

[2] P. C. Hargrave, G. Savini, N. Trappe, A. Challinor, S. B. Sørensen, P. A. R. Ade, R. V. Sudiwala, I. K. Walker, M. Gradziel, N. Tynan, and M. van der Vorst, "Coated dielectric lens design, modeling and measurements for future CMB polarimetry missions," in Proc. EuCAP, Gothenburg, Sweden, 2013.

[3] GRASP: General Reflector Analysis Program, TICRA, Laderstrade 34, Copenhagen, Denmark, www.ticra.com., 2014.

[4] S. B. Sørensen and K. Pontoppidan, "Lens analysis methods for quasioptical systems," in 2nd European Conference on Antennas and Propagation (EuCAP 2007), Edinburgh, Nov. 2007.

[5] J. R. Mautz and R. F. Harrington, "H-field, E-field, and combined field solutions for bodies of revolution," Syracuse University, NY, USA, Tech. Rep. TR-77-2, Feb. 1977.

[6] A. A. Kishk and L. Shafai, "Different formulations for numerical solution of single or multibodies of revolution with mixed boundary conditions," IEEE Transactions on Antennas and Propagation, vol. 34, no. 5, pp. 666-673, 1986. 